\title{
ON THE LIMITS OF REAL VARIANTS
}

\author{
By J. Mercer. \\ [Received November 26th, 1906.-Read December 13th, 1906.]

\section{Introduction.}

There are two well known theorems due to Cauchy which are concerned with the limits of variants* (i.e., functions of a variable positive integer $n$ ) for infinite indices. The first + is to the effect that, if $X_{n}$ is any variant such that

$$
X_{n+1}-X_{n}
$$

tends to a definite limit $\lambda$ when $n$ is increased indefinitely, then

$$
n^{-1} X_{n}
$$

tends to the same limit. The second $\ddagger$ shows that, if $X_{n}$ is a positive variant such that

$$
X_{n+1} / X_{n}
$$

tends to a definite limit $\lambda$ when $n$ is increased indefinitely, then

tends to that limit.

$$
X_{n}^{1 / n}
$$

Now the converse of neither of these theorems is necessarily true.

To take a particular example, suppose that $X_{n}=a^{n} \theta(n)$ where $\theta(n)=n$ for odd values of $n$ and $=\frac{1}{2} n$ for even values.

Then

$$
1 \leqslant \theta(n) \leqslant n
$$

for all values of $n$. Consequently

$$
1 \leqslant \vdots \theta(n)\}^{1, n} \leqslant n^{1, n}
$$

Since $\operatorname{Lt}_{n=\infty} n^{1 / n}=1$, it is clear that

$$
\left.\operatorname{Lt}_{n=\infty} ; a^{n} \theta(n)\right\}^{1 i n}=a .
$$

But $\operatorname{Lt}_{n=\infty} X_{n+1} / X_{n}$ does not exist, since

$$
\operatorname{Lt}_{n=\infty} X_{2 n+1} / X_{2 n}=2 a \text { and } \operatorname{Lt}_{n=\infty} X_{2 n} / X_{2 n-1}=\frac{1}{2} a \text {. }
$$

- Cf. Meray, Nonveau Précis d'Analyse infinitésimale, p. 1.

† "Cours d'Analyse de l'Ecole royale polytechnique" (1821), EGuvres Complates, nme. Série, t. nix., p. 54-58, p. 62-63.

† Cauchy, loc. cit., pp. 51-63. 
Examples such as this suggest the question: Can we find some single variant analogous to the $X_{n+1}-X_{n}$ or $X_{n+1} / X_{n}$ of Cauchy's theorems such that the existence of its limit $f_{o r}$ an infinite index will ensure the existence of both

$$
\operatorname{Lt}_{n=\infty}\left(X_{n+1}-X_{n}\right), \quad \operatorname{Lt}_{n=\infty} n^{-1} X_{n} \text { or } \operatorname{Lt}_{n=\infty} X_{n+1} / X_{i}, \quad \operatorname{Lt}_{n=\%} X_{n}^{1 / n}
$$

as the case may be? This will be considered in Section $I^{*}$ The succeeding section will be devoted to the extension of the theorems to the case of variants of more than one index. Section III. will be devoted to an extension of Pincherle's theorem on the radius of convergence of a power series which arises natually from the analysis of the first section.

1. We are to shew that there do exist single functions answering the above mentioned requirements. In fact, analogous to Cauchy's first theorem, we have the followingt:-

Theorem I.-Let $X_{n} \ddagger$ be any variant whose index is $n$ and which is such that

$$
X_{n+1}-X_{n}+\mu n^{-1} X_{n}
$$

tends to a definite limit $\lambda$ when $n$ tends to infinity; then (i.), if $\lambda$ is finite,

$$
X_{n+1}-X_{n} \text { and } n^{-1} X_{n}
$$

tend simultaneously to the limit $\lambda /(\mu+1)$, provided that $\mu+1>0$; (ii.) if $\lambda$ is infinite $( \pm \infty), n^{-1} X_{n}$ tends to $\lambda$ when $\mu+1>0$, whilst $X_{n+1}-X_{n}$ certainly does so only if $0 \geqslant \mu>-1$.

It will be observed that this theorem when proved will give us a single limit whose existence is necessary and sufficient for the two limits $\operatorname{Lt}_{n=\infty} X_{n+1}-X_{n}$ and $\operatorname{Lt}_{n=0} n^{-1} X_{n}$ so long as they are finite.

We now proceed to prove it. Suppose, in the first place, that $\lambda$ is finite. By the definition of limit we can, if $\epsilon$ is any arlitrurily assigned positive quantity, however small, find an integer $m$ such that, if $n \geqslant m$, we have

$$
\lambda-\sigma<X_{n+1}-X_{n}+\mu n^{-1} X_{n}<\lambda+\sigma
$$

where $\sigma=\frac{1}{2}(\mu+1) \epsilon, \sigma$ being positive since $\mu+1>0$.

Writing $n=m$ in this equality and dividing along by $(1-\mu / m)$ (which can be taken to be positive, since we may always suppose $m$

- Where it will be answered in the affirmative.

+ Of course, the theorem may be stated in various forms : e.!!, we maly write

$$
x_{n}=s_{1}+s_{2}+\ldots+s_{n}
$$

$\mp$ It is assumed that $x_{n}$ is fiuite for finite valu 
so chosen as to be greater than $\mu$ ), we obtain

$$
\frac{\lambda-\sigma}{1-\frac{\mu}{m}}<\frac{X_{m+1}}{1-\frac{\mu}{m}}-X_{n}<\frac{\lambda+\sigma}{1-\frac{\mu}{m}} .
$$

Writing now $n=m+1$ in (i.) and dividing along by

$$
\left(1-\frac{\mu}{m}\right)\left(1-\frac{\mu}{m+1}\right)
$$

we have $\frac{\lambda-\sigma}{\left(1-\frac{\mu}{m}\right)\left(1-\frac{\mu}{m+1}\right)}<\frac{X_{n+2}}{\left(1-\frac{\mu}{m}\right)\left(1-\frac{\mu}{m+1}\right)}-\frac{X_{m+1}}{1-\frac{\mu}{m}}$

$$
<\frac{\lambda+\sigma}{\left(1-\frac{\mu}{m}\right)\left(1-\frac{\mu}{m+1}\right)} \text {. }
$$

Proceeding in this manner, we obtain $p$ double inequalities, of which the last is

$$
\frac{\lambda-\sigma}{\prod_{i=0}^{n-1}\left(1-\frac{\mu}{m+t}\right)}<\frac{X_{m+p}}{\prod_{t=0}^{p-1}\left(1-\frac{\mu}{m+t}\right)}-\frac{X_{n+t^{-1}}}{\prod_{t=0}^{p-2}\left(1-\frac{\mu}{m+t}\right)}<\frac{\lambda+\sigma}{\prod_{t=0}^{p-1}\left(1-\frac{\mu}{m+t}\right)} .
$$

If we add all these inequalities, we obtain

$$
(\lambda-\sigma) S_{p-1}<\frac{X_{m+\eta}}{\prod_{t=0}^{p-1}\left(1-\frac{\mu}{m+t}\right)}-X_{m}<(\lambda+\sigma) S_{p-1}
$$

where $S_{p-1}$ is the sum of the series

$$
\frac{1}{1-\frac{\mu}{m}}+\frac{1}{\left(1-\frac{\mu}{m}\right)\left(1-\frac{\mu}{m+1}\right)}+\ldots+\frac{1}{\prod_{t=0}^{p-1}\left(1-\frac{\mu}{m+t}\right)} .
$$

Now, by a well known theorem of elementary algebra, ${ }^{*}$ the sum of this series is $(\mu+1 \neq 0)$

$$
\frac{1}{\mu+1}\left\{\frac{m+p}{\prod_{t=0}^{p-1}\left(1-\frac{\mu}{m+t}\right)}-m\right\} .
$$

Consequently, multiplying along the equality (ii.) by

$$
\frac{\operatorname{II~}_{t=0}^{p-1}\left(1-\frac{\mu}{m+t}\right)}{m+p},
$$

- Cf. A Trealise on Algebra, C. Smith, Fourth Edition (1893), p. 410. 
after having added $X_{n}$ to each term of it ; we obtain

$$
\begin{aligned}
\frac{\lambda-\sigma}{\mu+1}+\left\{\frac{\left(\prod_{t=0}^{\prime \prime 1}\left(1-\frac{\mu}{m+t}\right)\right.}{m+p}\right\} ; X_{m}-\frac{m(\lambda-\sigma) !}{\mu+1} ; \\
\quad<\frac{X_{m+i}}{m+p}<\frac{\lambda+\sigma}{\mu+1}+\left\{\frac{\prod_{t=0}^{p-1}\left(1-\frac{\mu}{m+t}\right)}{m+p}\right\}\left\{X_{m}-\frac{m(\lambda+\sigma) !}{\mu+1}\right\}
\end{aligned}
$$

Now, provided that $\mu+1>0$, the expression

$$
\frac{\prod_{t=0}^{p-1}\left(1-\frac{\mu}{m+t}\right)^{*}}{m+r}
$$

tends to zero $t$ when $p$ is increased indefinitely: we can therefore choose a value of $p$-say, $P$-such that $p \geqslant P$; the second members of the extreme terms of this last inequality are less than $\frac{1}{2} \epsilon$ in absolute magnitude. We have then, on writing $m+p=n, m+P=N$,

$$
\frac{\lambda}{\mu+1}-\epsilon<\frac{X_{n}}{n}<\frac{\lambda}{\mu+1}+\epsilon
$$

provided that $n \geqslant N$.

'l'his proves that $n^{-1} X_{n}$ tends to the limit $\lambda /(\mu+1)$ as $n$ increases indefinitely.

From this it follows at once, by well known theorems on limits, that

$$
\begin{aligned}
\operatorname{Lt}_{n=\infty}\left(X_{n+1}-X_{n}\right) & =\operatorname{Lt}_{n=\infty}\left(X_{n+1}-X_{n}+\mu \frac{X_{n}}{n}\right)-\mu \operatorname{Lt}_{n=\infty} \frac{X_{n}}{n} \\
& =\lambda-\frac{\lambda \mu}{\mu+1}=\frac{\lambda}{\mu+1},
\end{aligned}
$$

which completes the proof of the theorem for the case where $\lambda$ is finite.

2. Next, suppose that $\lambda=+\infty$.

Let $H$ be any positive number as great as we please. Then, corresponding to (i.) above, we have the single inequality

$$
X_{n+1}-X_{n}+\mu n^{-1} X_{n}>H(\mu+1)+\sigma \quad(n \geqslant m),
$$

$\sigma$ being a positive number.

Corresponding to (ii.), we have

$$
\frac{X_{m+p}}{\prod_{,=n}^{n-1}\left(1-\frac{\mu}{m+t}\right)}-X_{n n}>[H(\mu+1)+\sigma] S_{p-1} ;
$$

- Hereafter to be denoted oy $\eta_{\mu}$.

SER. 2. VOL. 5. No. $95 \%$.

+ Lif. C. Smit., op. cit., pp. $423-4$. 
whilst, instead of (iv.), we have

$$
\frac{X_{m+p}}{m+p}>H+\frac{\sigma}{\mu+1}+S_{p}
$$

where $S_{p}$, tends to zero when $p$ is increased indefinitely. Consequently, choosing $P$ so great that for $p \geqslant P$ we have $\left|S_{p}\right|<\sigma /(\mu+1)$, we deduce, on writing $m+p=n, m+P=N$,

$$
n^{-1} X_{n}>H \quad(n \geqslant N),
$$

which proves that

$$
\operatorname{Lt}_{n=\infty} n^{-1} X_{n}=+\infty \text {. }
$$

It follows, then, at once that

$$
\operatorname{Lt}_{n=\infty}\left(X_{n+1}-X_{n}\right)=\operatorname{Lt}_{n=\infty}\left[X_{n+1}-X_{n}+\mu n^{-1} X_{n}\right]-\mu \operatorname{Lt}_{n=\infty} n^{-1} X_{n}=+\infty,
$$

if $\mu \leqslant 0$. The remaining case, viz. $\lambda=-\infty$, may at once be deduced (by writing $X_{n}=-X_{n}^{\prime}$ ) from the preceding.

The theorem is then completely established.

3. As corollaries of Theorem I. we have :

Cor. I.-If we write $\mu=0$ in the above, we at once obtain Cauchy's theorem.

Cor. II.-Suppose that $X_{n}$ is such that $n^{-2} X_{n}$ tends to zero when $n$ becomes indefinitely great.

The theorem may be then stated in the form :

If $X_{n}$ be any variant such that $n^{-2} X_{n}$ tends to zero when $n$ increases indefinitely, whilst at the same time

$$
n^{-\mu}\left\{n^{\mu} X_{n+1}-(n-1)^{\mu} X_{n}\right\}
$$

tends to a definite limit $\lambda$, then, $\mu+1>0$, we have

$$
\operatorname{Lt}_{n=\infty}\left(X_{n+1}-X_{n}\right)=\operatorname{Lt}_{n=\infty} \frac{X_{n}}{n}=\frac{\lambda}{\mu+1} \text {. }
$$

As a simple application of this corollary we may evaluate

$$
\operatorname{Lt}_{n=\infty} \frac{1^{\mu}+2^{\mu}+\ldots+n^{\mu}}{n^{\mu+1}} \quad(\mu+1>0) .
$$

Write

$$
X_{n+1}=\frac{1^{\mu}+2^{\mu}+\ldots+n^{\mu}}{n^{\mu}} \text {. }
$$

Obviously, if $\mu>0$,

$$
X_{n+1}<n
$$

if $\mu<0$,

$$
X_{n+1}<n^{1-\mu} \text {. }
$$


Consequently, $\mu+1>0$, the $\operatorname{Lt}_{n=\infty} n^{-2} X_{n}=0$.

Again, $\quad n^{\mu} X_{n+1}-(n-1)^{\mu} X_{n}=n^{\mu}$.

We have therefore in this case $\lambda=1$; therefore

$$
\operatorname{Lt}_{n=\infty} \frac{X_{n}}{n}=\operatorname{Lt}_{n=\infty} \frac{1^{\mu}+2^{\mu}+\ldots+n^{\mu}}{n^{\mu+1}}=\frac{1}{\mu+1} \cdot *
$$

4. Before proceeding further, let us examine the limitation (viz., $\mu+1>0$ ) which has been forced upon us in the course of the proof when $\lambda$ is finite.

It will be observed that the proof turns upon the fact that

$$
\operatorname{Lt}_{p=\infty} \frac{\prod_{t=0}^{p-1}\left(1-\frac{\mu}{m+t}\right)}{m+p}
$$

tends to zero when $p$ is increased indefinitely, provided $\mu+1>0$. If $\mu+1 \leqslant 0$, our argument breaks down, and, if the theorem is true for a wider range of values of $\mu$, we should have to find some new line of reasoning.

It will be seen, however, by the following particular example, that it is impossible to widen the range of values of $\mu$ in the general case.

Let

$$
X_{n+1}=(1-\mu)\left(1-\frac{\mu}{2}\right) \ldots\left(1-\frac{\mu}{n}\right) \text {. }
$$

Then we have always $X_{n+1}-\left(1-\frac{\mu}{n}\right) X_{n}=0$.

But

$$
\frac{X_{n+1}}{n+1}=\frac{(1-\mu)\left(1-\frac{\mu}{2}\right) \ldots\left(1-\frac{\mu}{n}\right)}{(1+1)\left(1+\frac{1}{2}\right) \ldots\left(1+\frac{1}{n}\right)},
$$

which tends to the limits $0,1, \infty$ according as $\mu+1>$, =, or $<0$.

This shows at once that $\mu+1>0$ is the greatest range of values for which the theorem is generally true.

5. We have next to inquire whether in the case $\lambda= \pm \infty$ we could hope to prove generally that $\operatorname{Lt}_{n=\infty}\left(X_{n+1}-X_{n}\right)=\lambda$ when $\mu>0$. As in the preceding, we shall prove that this is impossible by a particular example.

* In the language of the integral calculus $\int_{0}^{1} x \mu d x=\frac{1}{\mu+1}(\mu+1>0)$, it is eiling to see how to evaluate directly such integrals as $\int_{0}^{1} \int_{0}^{x} x^{\mu \nu} d x d y(\nu+1>0, \mu+\nu+2>0)$ ! 
Let a variant be defined by the following :-

Obviously

$$
X_{2 n+1}=\frac{1}{2} n(n+3), \quad X_{2 n}=\frac{1}{2} n(n+1) .
$$

But

$$
X_{2_{n+1}}-X_{2 n}=n, \quad X_{2 n}-X_{!_{n-1}}=1 \text {. }
$$

$$
\operatorname{Lt}_{n=\infty}\left\{X_{n+1}-X_{n}+\mu n^{-1} X_{n}\right\}=+\infty \quad(\mu>0),
$$

whilst the two equations just given above show that $X_{n+1}-X_{n}$ oscillates between 1 and $+\infty$.

It is easy to see, however, that, if we regard the numbers of the sequence $X_{n+1}-X_{n}$ as represented by points on a line, even if $\mu>0, \lambda$ is a limiting point of the set.

6. Before leaving this theorem we may remark that the condition

$$
\operatorname{Lt}_{n=\infty}\left(X_{n+1}-X_{n}+\mu n^{-1} X_{n}\right)=\lambda \quad(\mu+1>0)
$$

is necessary and sufficient for the existence of the limits

$$
\operatorname{Lt}_{n=\infty}\left(X_{n+1}-X_{n}\right) \text { and } \operatorname{Lt}_{n=\infty} n^{-1} X_{n}
$$

only if $\lambda$ is finite. If $\lambda$ is infinite, the above condition is necessary, if $\mu>0$, but is not sufficient; whilst, if $\mu<0$ but $\mu+1>0$, the condition is sufficient, but not necessary. This last point may be made clear by the following example:-

Let $\mu$ be a negative number $(>-2), r$ a positive number $=-\frac{4}{\mu}-1$, and let $a$ variant be defined by the equations

$$
X_{2 n+1}-X_{2 n}=m, \quad X_{2 n}-X_{2 n-1}=n \text {; }
$$

so that $\quad X_{2 n+1}=\frac{(1+r) n(n+1)}{2}, \quad X_{2 n}=\frac{m(n-1)}{2}+\frac{n(n+1)}{2}$.

Then, obviously, $\operatorname{Lt}_{n=\infty}\left(X_{n+1}-X_{n}\right)=\infty, \quad \operatorname{Lt}_{n=\infty} n^{-1} X_{n}=\infty$;

but

$$
\begin{aligned}
\operatorname{Lt}_{n=\infty}\left[X_{2 n+1}-X_{2 n}+\mu \frac{X_{2 n}}{2 n}\right] & =+\infty \\
\operatorname{Lt}_{n=\infty}\left[X_{2 n}-X_{2 n-1}+\mu \frac{X_{2 n-1}}{2 n-1}\right] & =\text { finite quantity. }
\end{aligned}
$$

7. It is easy to see that, by a slight modification of the above argument, we can generalise this theorem." Let $f(x)$ be a function of $x$ defined for all real values of $x$ greater than a fixed number and

* Cf. Cauchy, op. cit., pp. 54-58. 
which is finite for finite values of $x$. The theorem referred to is the following :-

Theorem II.-If for increasing values of $x$ the difference

$$
f(x+1)-\left(1-\frac{r}{x}\right) f(x)
$$

tends to the limit $\lambda$, then the functions

$$
f(x+1)-f(x) ; \quad x^{-1} f(x)
$$

tend at the same time to the limit $\lambda /(r+1)$, provided that $\lambda$ is finite and $r+1>0$ : in the case when $\lambda= \pm \infty$ these each tend to $\lambda$ if $0 \geqslant r>-1$; whilst, if $r>0, \operatorname{Lt}_{n=\infty} x^{-1} f(x)=\lambda$, but $f(x+1)-f(x)$ may not tend to this limit.

8. Corresponding to Cauchy's second theorem, we have the following :-

Theorem III.-Let $X_{n}$ be a variant, positive for great enough values of $n$, such that

$$
\frac{X_{n+1}}{X_{n}^{(1-\mu / n)}}
$$

tends to a definite limit $\lambda$ when $n$ increases indefinitely; then

$$
X_{n+1} / X_{n} \text { and } X_{n}^{1 / n}
$$

each tends to the limit $\lambda^{1 /(n+1)}$, if $\lambda$ is finite and not zero and $\mu+1>0$, whilst, if $\lambda=0$ or $\infty$, each tends to the limit $\lambda$, provided that $0 \geqslant \mu>-1$. In the latter case $X_{n}^{1 / n}$ certainly tends to the limit $\lambda$ if $\mu>0$, though $X_{n+1} / X_{n}$ may not do so.

As before, we may remark that this will give a single limit whose existence is necessary and sufficient for that of two-in this case

$$
\operatorname{Lt}_{n=\infty} X_{n+1} / X_{n} \text { and } \operatorname{Ltt}_{n=\infty} X_{n}^{1 / n}
$$

-provided $\lambda$ is neither zero nor infinite.

It is, of course, quite clear that Theorem III. may be deduced from Theorem I. by writing $\log X_{n}^{\prime}=X_{n}$; but, following Cauchy's lead in his proof of the classical theorems, a separate proof which does not require the theory of logarithms may be supplied. Space does not, however, permit such proof being given here. The reader will have little difficulty in constructing it if he so desires. 
Corresponding to Theorem II., it is easily seen that we have the following generalisation of Theorem III. :-

Theorem IV.-If for increasing values of $x$ the quotient

$$
\frac{f(x+1)}{[f(x)]^{1-r / 2}}
$$

tends to the definite limit $\lambda$, then (1), if $\lambda$ is finite and not zero, the functions

$$
[f(x+1) / f(x)] \text { and }[f(x)]^{1 / x} \text {. }
$$

tend at the same time to the limit $\lambda^{1 /(r+1)}(r+1>0)$; (2), if $\lambda=\infty$ or 0 , these each tend to $\lambda$ if $0 \geqslant r>-1$; whilst, if $r>0, \operatorname{Lt}_{r=\infty}[f(x)]^{1 / x}=\lambda$, though $f(x+1) / f(x)$ may not tend to this limit.

II.

9. In extending the theorems of I. to variants of more than one index we will confine ourselves to the consideration of the case when there are only two, as the statement of the theorems and their proof will be sufticiently obvious in the more complex cases.

Corresponding to Theorem I. of the previous section, we have:

Theorem I.-Let $X_{m, n}$ be a variant of two positive indices $m$ and $n$, such that $\left(X_{m+1, n}-X_{m, n}\right)$ is limited* for $m$ infinite and $\left(X_{m, n+1}-X_{m, n}\right)$ for $n$ infinite; moreover, suppose that for increasing values of $m$ and $n$ the variant

$$
X_{m+1, n+1}-\left(1-\frac{\mu}{m}\right) X_{m, n+1}-\left(1-\frac{\nu}{n}\right) X_{m+1, n}+\left(1-\frac{\mu}{m}\right)\left(1-\frac{\nu}{n}\right) X_{m, n}
$$

tends to a definite limit $\lambda$. Then

(i.) If $\lambda$ is finite,

The variants $X_{m+1, n+1}-X_{m, n+1}-X_{m+1, n}+X_{m, n}$,

$$
\frac{X_{m+1, n}-X_{m, n}}{n}, \quad \frac{X_{m, n+1}-X_{m, n}}{m}, \quad \frac{X_{n, n}}{m n}
$$

all tend to the limit $\uparrow$

$$
\frac{\lambda}{(\mu+1)(\nu+1)},
$$

- A variant $X_{m, n}$ is limited for $m$ infinite if for any finite value of $n$ we can find two finite numbers $l_{n}$ and $L_{n}$ such that $l_{n}<X_{m, n}<L_{n}$ for all values of $m$. It is easy to see that, if $X_{m+1, n}-X_{m, n}$ is so limited, then $m^{-1} X_{m, n}$ is also limited.

+ To remove all possibility of doubt, it may be stated that here and thronghout a variant of two indices $X_{m, n}$ will be said to tend to a finite number $\lambda$ for increasing values of $m$ and $n$, when or any assigned positive number $\epsilon$ we can find integers $M$ and $N$ so that $\left|X_{m, n}-\lambda\right|<\epsilon$, provided $m \geqslant M$ and $n \geqslant N$. Should $\lambda$ be infinite, the definition is modified in the usual manner. 
when $m$ and $n$ are increased indefinitely, provided that $\mu+1>0$, $\nu+1>0$.

(ii.) If $\lambda= \pm \infty$,

(a) The last of the above variants tends to $\lambda$, provided that

$$
\mu+1>0, \quad \nu+1>0 \text {. }
$$

(b) The third variant tends to $\lambda$, provided that

$$
0>\nu>-1, \quad \mu+1>0 .
$$

(c) The second tends to $\lambda$, provided that $0>\mu>-1, \nu+1>0$.

(d) The first variant tends to $\lambda$, provided that $\mu$ or $\nu=0$, and the one $\neq 0$ lies between 0 and -1 .

We remark that this theorem, when proved, will give us a single variant the existence of whose (finite) limit for $m$ and $n$ indefinitely increased is the necessary and sufficient condition that the four variants mentioned above should have (finite) limits.

10. We proceed to prove the theorem, assuming in the first place that $\lambda$ is finite.

Consider the last variant, viz., $m^{-1} n^{-1} X_{m, n}$. Then, if $\epsilon$ be any arbitrarily assigned positive number, however small, we can under the proposed conditions find two integers $m$ and $n$, such that when $N \geqslant n$, $M \geqslant m$, we have

$$
\begin{aligned}
\lambda-\sigma<\left[X_{M+1, N+1}-\left(1-\frac{\mu}{M}\right)\right. & X_{M, N+1}-\left(1-\frac{\nu}{N}\right) X_{M+1, N} \\
& +\left(1-\frac{\mu}{M}\right)\left(1-\frac{\nu}{N}\right) X_{M, N]}<\lambda+\sigma
\end{aligned}
$$

where

$$
\sigma=\frac{1}{2}(\mu+1)(\nu+1) \epsilon \text {. }
$$

Writing now $N=n, M=m$, and dividing through the inequality by $\left(1-\frac{\mu}{m}\right)\left(1-\frac{\nu}{n}\right)$ (which may be assumed positive, since we can always assume $m$ and $n$ chosen greater than $\mu$ and $\nu$ respectively), we have

$$
\begin{aligned}
\frac{\lambda-\sigma}{\left(1-\frac{\mu}{m}\right)\left(1-\frac{\nu}{n}\right)} & <\left\{\frac{X_{m+1, n+1}}{\left(1-\frac{\mu}{m}\right)\left(1-\frac{\nu}{n}\right)}-\frac{X_{m, n+1}}{\left(1-\frac{\nu}{n}\right)}-\frac{X_{m+1, n}}{\left(1-\frac{\mu}{m}\right)}+X_{m, n}\right\} \\
& <-\frac{\lambda+\sigma}{\left(1-\frac{\mu}{m}\right)\left(1-\frac{\nu}{n}\right)} .
\end{aligned}
$$


Again, writing $M=m+1, N=n$, and dividing through by

we obtain

$$
\left(1-\frac{\mu}{m}\right)\left(1-\frac{\mu}{m+1}\right)\left(1-\frac{\nu}{n}\right) \text {, }
$$

$$
\begin{aligned}
& \frac{\lambda-\sigma}{\left(1-\frac{\mu}{m \prime}\right)\left(1-\frac{\mu}{m-1}\right)\left(1-\frac{\nu}{n}\right)} \\
& \left.\quad \vdots \frac{X_{m+2, n+1}}{\left(\prod_{t=0}^{1}\left(1-\frac{\mu}{m+t}\right)\left(1-\frac{\nu}{n}\right)\right.}-\frac{X_{m+1, n+1}}{\left(1-\frac{\mu}{m}\right)\left(1-\frac{\nu}{n}\right)} \cdots\right\} \\
& \quad<\frac{\lambda+\sigma}{\left(1-\frac{\mu}{m}\right)\left(1-\frac{\mu}{m+1}\right)\left(1-\frac{\nu}{n}\right)} .
\end{aligned}
$$

Proceeding in this manner and giving $M$ all possible values $<m+p$, and $N$ all possible values $<n+q$, we have a number of inequalities of which the last is

$$
\begin{aligned}
\frac{\lambda-\sigma}{\prod_{t=0}^{n-1}\left(1-\frac{\mu}{m+t}\right) \prod_{t^{\prime}=0}^{1-1}\left(1-\frac{\nu}{n+t^{\prime}}\right)} & <\frac{X_{m+\mu^{\prime}, n+1}}{\prod_{t=0}^{n-1}\left(1-\frac{\mu}{m+t}\right) \prod_{t^{\prime}=0}^{\prime-1}\left(1-\frac{\nu}{n+t}\right)} \cdots \\
& <\frac{\lambda+\sigma}{\prod_{t=0}^{p-1}\left(1-\frac{\mu}{m+t}\right) \prod_{t^{\prime}=0}^{\prime-1}\left(1-\frac{\nu}{n+t^{\prime}}\right)}
\end{aligned}
$$

Adding all these inequalities, we obtain

$$
\begin{aligned}
& (\lambda-\sigma) S_{l^{\prime}-1,1,-1}<! \frac{X_{n+p, n+\ell}}{! \prod_{l=4}^{n-1}\left(1-\frac{\mu}{m+t}\right) \prod_{t^{\prime}=0}^{q-1}\left(1-\frac{\nu}{n+t}\right)}-\frac{X_{m, n+\imath}}{\prod_{t^{\prime}=0}^{n-1}\left(1-\frac{\nu}{n+t^{\prime}}\right)} \\
& \left.-\frac{Y_{i t+p, n}}{\prod_{t=0}^{p-1}\left(1-\frac{\mu}{m+t}\right)}+X_{m, n}\right\} \\
& <(\lambda+\sigma) S_{p-1, y-1}
\end{aligned}
$$$$
\text { where } \quad S_{p-1, q-1}=\sum_{s=0}^{p-1} \sum_{s^{\prime}=0}^{\eta-1} \frac{1}{\prod_{t=0}^{s}\left(1-\frac{\mu}{m+t}\right) \prod_{t^{\prime}=0}^{s}\left(1-\frac{\nu}{n+t^{\prime}}\right)} \text {. }
$$

Now it is clear that the sum of this series is equal to

$$
\sum_{s=0}^{\prime \prime-1} \frac{1}{\prod_{t=0}^{s}\left(1-\frac{\mu}{m+t}\right)} \cdot \sum_{s^{\prime}=0}^{\prime-1} \frac{1}{\prod_{t^{\prime}=0}^{s}\left(1-\frac{\nu}{n+t^{\prime}}\right)} .
$$


Consequently, if we denote by $\eta_{p}$ the same quantity as in the previous section, and by $\xi_{4}$ the corresponding quantity when $n$ is written for $m$ and $q$ for $p$, we have after an easy reduction

$$
\begin{aligned}
& \frac{\lambda-\sigma}{(\mu+1)(\nu+1)}\left(1-m \eta_{p}\right)\left(1-n \xi_{1}\right)+\frac{X_{m, n+q}}{n+q} \eta_{p}+\frac{X_{m+\mu_{1}, n}}{m+p} \xi_{q}-X_{m, n} i_{1} \dot{\zeta}_{\eta} \\
& <\frac{X_{n+p, n+q}}{(m+p)(n+q)}<\frac{\lambda+\sigma}{(\mu+1)(\nu+1)}\left(1-m \eta_{p}\right)\left(1-n \dot{\xi_{1}}\right) \\
& +\frac{Y_{m, n+1,}}{n+q} \eta_{l}+\frac{X_{m+1, n}}{m+p}-X_{i n, n} \eta_{p} \xi_{i} \text {. }
\end{aligned}
$$

Now it is obvious* that when $p$ and $q$ increase indefinitely the left-and right-hand sides of this double inequality tend to the limits $\begin{gathered}\lambda-\sigma \\ (\mu+1)(\nu+1)\end{gathered}$ and $\frac{\lambda+\sigma}{(\mu+1)(\nu+1)}$ respectively, i.e., to the limits $\frac{\lambda}{(\mu+1)(\nu+1)}-\frac{\epsilon}{2}$ and $\frac{\lambda}{(\mu+1)(\nu+1)}+\frac{\epsilon}{2}$. It is possible therefore to choose two positive integers $P$ and $Q$ such that

$$
\frac{\lambda}{(\mu+1)(\nu+1)}-\epsilon<\frac{X_{m+\beta, n+1}}{(m+p)(n+q)}<\frac{\lambda}{(\mu+1)(\nu+1)}+\epsilon,
$$

provided that $p \geqslant P, q \geqslant Q$. This is sufficient to show that

$$
\operatorname{Lt}_{m=\infty, n=\infty} \frac{X_{m, n}}{m n}=\frac{\lambda}{(\mu+1)(\nu+1)} \text {. }
$$

11. Now let us consider the third variant. The number $\sigma$ used in the inequality $(\alpha)$ is quite at our disposal, though, of course, $m$ and $n$ vary with it. The number $\epsilon$ being as before, choose $\sigma=\frac{1}{2}(\mu+1) \epsilon$. Taking those of the inequalities $(\beta)$ for which $q=1$ and adding them, we obtrin

$$
\begin{aligned}
& \frac{\lambda-\sigma}{\left(1-\frac{\nu}{n}\right)} S_{p-1} \\
& \quad<\left\{\frac{X_{m+1, n+1}}{\left(1-\frac{\nu}{n}\right) \prod_{t=0}^{p-1}\left(1-\frac{\mu}{m+t}\right)}-\frac{X_{m+\mu, n}}{\prod_{t=0}^{p-1}\left(1-\frac{\mu}{m+t}\right)}-\frac{X_{m, n+1}}{\left(1-\frac{\nu}{n}\right)}+X_{m, n}\right\} \\
& \quad<\frac{\lambda+\sigma}{\left(1-\frac{\nu}{n}\right)} S_{p-1} .
\end{aligned}
$$

- Since $\underset{p=\infty}{\mathrm{Lt}} \eta_{p}=0, \underset{q=\infty}{\mathrm{Lt}} \zeta_{\eta}=0$, and in virtue of the conditions of limitedness imposed in tise variant. 
Using the notation of I., $\$ 1$, we obtain

$$
\begin{aligned}
\frac{(\lambda-\sigma)}{\mu+1}\left(1-m \eta_{\eta}\right)+\left[X_{m, n+1}-X_{m, n}\right] \eta_{1}+\frac{\nu}{n} X_{m, n} \eta_{p}-\nu \frac{X_{m+\eta, n}}{(m+p) n} \\
<\frac{X_{m+p_{, n+1}-X_{m+p, n}}}{m+p}<\frac{\lambda+\sigma}{\mu+1}\left(1-m \eta_{p}\right)+\ldots
\end{aligned}
$$

Now consider the terms on the left-hand side when $p$ and $n$ increase indefinitely. The first tends to the limit $(\lambda-\sigma) /(\mu+1)$; the second and third vanish in virtue of the conditions of limitedness imposed; the fourth tends to the limit

$$
\frac{\lambda \nu}{(\mu+1)(\nu+1)}
$$

in virtue of the result proved in the preceding paragraph. Similarly for terms on the right.

Recalling that $\sigma /(\mu+1)=\frac{1}{2} \epsilon$, it follows that we can choose two positive integers $P$ and $N$ such that, if $p \geqslant P$ and $n \geqslant N$, we have

$$
\frac{\lambda}{(\mu+1)(\nu+1)}-\epsilon<\frac{X_{m+p_{1} n+1}-X_{m+\psi_{2}, \mu}}{m+p}<\frac{\lambda}{(\mu+1)(\nu+1)}+\epsilon .
$$

This is sufticient to prove that

$$
\operatorname{Lt}_{m=\infty, n=\infty} \frac{X_{m, n+1}-X_{m, n}}{m}=\frac{\lambda}{(\mu+1)(\nu+\mathrm{j} .)} .
$$

In the same manner we may prove that the second variant tends to the same limit. It then follows at once by methods analogous to those used at the end of $\$ 1$ that the first variant tends to this limit. We have therefore proved the theorem for the case where $\lambda$ is finite. The proof of the second part of the theoren we do not propose to give: the reader will have no difficulty in constructing it, in view of what has just been done here and in $\$ 2$ above.

12. We have the following corollaries of this theorem :-

Cor. I. -Writing $\mu=\nu=0$, we have the two-dimensional analogue of Cauchy's tirst theorem, viz.,

If for increasing values of $m$ and $n$ the variant

$$
X_{m+1, n+1}-X_{m, n+1}-X_{n+1, n}+X_{n, n}
$$


tends to a definite limit $\lambda$, then

$$
\frac{X_{m, n}}{m n}, \quad \frac{X_{m+1, n}-X_{n, n}}{n}, \quad \frac{X_{m, n+1}-X_{m, n}}{m}
$$

tend to the same limit.

The theorem may, of course, be stated in various ways, e.g., writing

we have it in a new form.

$$
X_{n, n}=\sum_{r=1}^{m} \sum_{s=1}^{n} S_{r s}
$$

Cor. II. - Let

$$
\operatorname{Lt}_{m=\infty, n=\infty} \frac{X_{m, n+1}-X_{n, n}}{m^{2}}=\operatorname{Lt}_{m=\infty, n=\infty} \frac{X_{m+1, n}-X_{m, n}}{n^{2}}=\operatorname{Lt}_{m=\infty, n=\infty} \frac{X_{m, n}}{n^{r} m^{s}}=0,
$$

the latter for all positive integer values of $r$ and $s, r=s=1$ excluded. We deduce at once-

If for increasing values of $m$ and $n$ the variant

$$
\begin{aligned}
& m^{-\mu} n^{-\nu}\left\{m^{\mu} n^{\nu} X_{m+1, n+1}-(m-1)^{\mu} n^{\nu} X_{m, n+1}-(n-1)^{\nu} m^{\mu} X_{m+1, n}\right. \\
& \text { tends to the definite limit } \lambda \text {, then } \\
& \text { then }
\end{aligned}
$$

$$
\operatorname{Lt}_{m=\infty, n=\infty} \frac{X_{m, n}}{m n}=\frac{\lambda}{(\mu+1)(\nu+1)} \quad(\mu+1>0, \nu+1>0) .
$$

As a simple example of this consider

$$
\text { Lt } \quad 1^{s}\left(1^{r}+2^{r}+3^{r}+\ldots+m^{r}\right)+2^{s}\left[1^{r}+2^{r}+\ldots+(2 m-1)^{r}+(2 m)^{r}\right]+\ldots
$$
$n=\infty, n=\infty$, $r+1>0$

$$
\frac{+n^{s}\left[1^{r}+2^{r}+\ldots+(n m-1)^{r}+(n m)^{r}\right] .}{n^{r+s+2} m^{r+1}} \text {. }
$$

If we write this equal to $\frac{X_{m+1, n+1}}{m n}$, it is easy to see that the above conditions are all fulfilled; moreover, we have

$$
\begin{aligned}
m^{r}\left[n^{r+s+1} X_{m+1, n+1}-(n-1)^{r+s+1}\right. & \left.X_{n+1, n}\right] \\
& =n^{s}\left\{1^{r}+2^{r}+\ldots+(n m-1)^{r}+n^{r} m^{r}\right\} .
\end{aligned}
$$

From which

$$
\begin{aligned}
m^{-r} n^{-(r+s+1)}\left\{m^{r} n^{r+s+1}\right. & \left.X_{m+1, n+1} \ldots\right\} \\
& =\frac{1+\left(1-\frac{1}{n m}\right)^{r}+\left(1-\frac{2}{n m}\right)^{r}+\ldots+\left(1-\frac{1}{m}\right)^{r}}{n} .
\end{aligned}
$$

Obviously this tends to the limit 1 when $m$ and $n$ are increased indefinitely. 
We see therefore that

$$
\operatorname{Lt}_{m=\infty, n=\infty} \frac{X_{m+1, n+1}}{(m+1)(n+1)}=\operatorname{Lt}_{m=\infty, n=\infty} \frac{X_{m, n}}{m n}=\frac{\lambda}{(r+1)(r+s+2)}, *
$$

provided, of course, $r+1$ and $r+s+2>0$.

13. Sufficient indications have now been given to show how the theorems of the preceding section extend to the case of variants of two indices. We will content ourselves therefore with enunciating the following theorems, ${ }^{+}$which are easily established :-

Theorem II.-If $f(x, y)$ is a real function of two positive variables $x$ and $y$ (defined for all values of $x>X$ and of $y>Y$ when $X$ and $Y$ are fixed numbers) such that $f(x+1, y)-f(x, y)$ is limited for $x>X, y \leqslant$ any finite number $>Y$, with a corresponding condition for $f(x, y+1)-f(x, y)$, then, if when $x$ and $y$ are increased indefinitely the function

$$
\begin{gathered}
f(x+1, y+1)-\left(1-\frac{r}{x}\right) f(x, y+1)-\left(1-\frac{s}{y}\right) f(x+1, y) \\
+\left(1-\frac{r}{x}\right)\left(1-\frac{s}{y}\right) f(x, y)
\end{gathered}
$$

tends to a finite limit $\lambda$,

$$
\begin{aligned}
\operatorname{Lt}_{x=\infty, y=\infty} \frac{f(x, y)}{x y} & =\operatorname{Lt}_{x=\infty, y=\infty} \frac{f(x+1, y)-f(x, y)}{y}=\operatorname{Lt}_{x=\infty, y=\infty} \frac{f(x, y+1)-f(x, y)}{x} \\
& =\operatorname{Lt}_{c=\infty, y=\infty}[f(x+1, y+1)-f(x, y+1)-f(x+1, y)+f(x, y)] \\
& \left.=\frac{\lambda}{(r+1)(s+1)} \quad \text { (provided } r+1>0, s+1>0\right) .
\end{aligned}
$$

Theorea III.-Let $X_{m, n}$ be a positive variant of two indices $m$ and $n$, such that $\left(\frac{X_{m+1, n}}{X_{m, n}}\right)^{ \pm 1}$ is limited for $m$ infinite, and $\left(\frac{X_{m, n+1}}{X_{m, n}}\right)^{ \pm 1}$ for $n$ infinite ; then, if for increasing values of $m$ and $n$ the variant

$$
\frac{X_{m+1, n+1} X_{m, n}^{(1-\mu / m)(1-\nu / n)}}{X_{m, n+1}^{(1-\mu, m)} X_{m+1, n}^{(1-\nu / n)}}
$$

- The limit evaluated is, of course, $\int_{0}^{1} d x \int_{0}^{x} x^{s} y^{\prime} d y$ for one particular method of dividing the field of integration into infinitesimal elements. An evaluation when the method of division is different (squares) is referred to in the footnote attached to the end of $\S 3$.

+ We only enunciate the theorems for the case in which $\lambda$ is finite, and in the last two theorems different from zero. There is no difficulty in stating them for other cases ( $f f$. Theorem of this section). 
tends to a definite finite limit $\lambda$ different from zero, the variants

$$
\frac{X_{n+1, n+1} X_{m, n}}{\bar{X}_{m, n+1} X_{m+1, n}}, \quad\left(\frac{X_{m+1, n}}{X_{m, n}}\right)^{1 ! n}, \quad\left(\frac{X_{m, n+1}}{X_{m, n}}\right)^{1 / m}, \quad\left(X_{m, n}\right)^{1 m n}
$$

tend simultaneously to the limit

$$
\lambda^{1 /(\mu+1)(\nu+1)},
$$

provided that $\mu+1>0, \nu+1>0$.

Cor. I.-Writing $\mu=\nu=0$, we have the two-dimensional analogue of Cauchy's second theorem.

Theorem IV.-If $f(x, y)$ is a real positive function of two variables $x$ and $y$ (defined for all values of $x>X$ and of $y>Y$, where $X, Y$ are fixed numbers), such that $[f(x+1, y) / f(x, y)]^{ \pm 1}$ is limited for $x>X$ and y $\leqslant$ any finite number $>Y$, with a corresponding condition for $[f(x, y+1) / f(x, y)]^{ \pm 1}$, then, if when $x$ and $y$ are increased indefinitely the function

$$
\frac{f(x+1, y+1)[f(x, y)]^{(1-r x)(1-s / y)}}{[f(x, y+1)]^{(1-r / x)}[f(x+1, y)]^{(1-s: y)}}
$$

tends to a finite limit $\lambda$ different from zero,

$$
\begin{aligned}
\operatorname{Lt}_{x=\infty, y=\infty} \frac{f(x+1, y+1) f(x, y)}{f(x, y+1) f(x+1, y)} & \\
= & \operatorname{Lt}_{x=\infty, y=\infty}\left(\frac{f(x, y+1)}{f(x, y)}\right)^{1 \ldots}=\operatorname{Lt}_{x=\infty, y=\infty}\left(\frac{f(x+1, y)}{f(x, y)}\right)^{1, y} \\
& =\operatorname{Ltt}_{x=\infty, y=\infty}[f(x, y)]^{1 / x y}=\lambda^{1 /(r+1)(s+1)},
\end{aligned}
$$

provided that $r+1>0, s+1>0$.

III.

14. The object of the present section is to prove an extension of Pincherle's well known theorem on the radius of convergence of a power series, and in some measure to bridge the gap between it and the CauchyHadamard theorem on the same subject. Pincherle's theorem" may be stated as follows :-

Let $a_{r}$ be the modulus of the coefficients of $z^{r}$ in $n$ nower series $P(z)$.

- Pincnerle, Lezioni sulla tcoriı delle finzioni aualitiche, Bologna, 1899-1900 (hthographed). See also Vivanti-Gutzmer, Theorie der eindentigen analytischen Funlitionen, Leipzig, 1906, p. 64. 
Then, if $L$ and $l$ be the greatest* and least† of the limits of the set of variants

$$
\frac{a_{1}}{a_{0}}, \frac{a_{2}}{a_{1}}, \ldots, \frac{a_{n+1}}{a_{n}}, \ldots
$$

the radius of convergence $(\rho)$ of the series satisfies the inequalities

$$
\begin{aligned}
& \rho \geqslant L^{-1}, \text { if } L \text { be finite, } \\
& \rho \leqslant l^{-1}, \text { if } l \neq 0 .
\end{aligned}
$$

15. Using the same convention with regard to $a_{r}$, the theorem we propose to prove is-

If $L$ and $l$ are respectively the greatest and least limits of the set of variants

$$
\frac{\alpha_{2}}{\alpha_{1}^{(1-r)}}, \frac{a_{3}}{\alpha_{2}^{(1-3 r)}}, \ldots, \frac{a_{n+1}}{\alpha_{n}^{(1-r / n)}}, \ldots, \quad(r+1>0),
$$

then the radius ( $\rho$ ) of convergence of the series satisfies the inequalities

(1) $\rho \geqslant L^{-1(r+1)}$, if $L$ is finite ;

(2) $\rho \leqslant l^{-1 .(r+1)}$, if $l \neq 0$.

Let the power series be

$$
P(z)=a_{0}+a_{1} z+a_{2} z^{2}+\ldots+a_{n} z^{n}+\ldots,
$$

so that $\left|a_{n}\right|=a_{n}$, and let $\xi=|z|$.

To prove (1) it will be sufficient to show that, if $\xi$ be any number $<1 / L^{1 /(n+1)}$, the above series is convergent; supposing this to be the case, let $K$ be a number such that

$$
\xi^{-(r+1)}>K>L
$$

Then, by hypothesis, the above mentioned set contains only a finite number of elements greater than $K$. We are able then to choose $m$ so that, if $n \geqslant m$,

$$
\frac{\alpha_{n+1}}{a_{n}^{(1-i / i)}} \gtrless K
$$

Writing $n=m$ in this inequality and supposing that $m$ is chosen $>r$, we have, on extracting the $(1-r / m)$-th root,

$$
\alpha_{m}^{-1} \alpha_{m+1}^{1 /(1-r m)}<K^{1(1-r m)}
$$

- "La plus grando des limites," Borel, Séries à termes positifs (1902), p. 9.

+ “La plus petite des limites," ibid., p. 10. 
Again, if we write $n=m+1$, and extract the $1 /\{(1-r / m)[1-r /(m+1)]\}$-th root, we obtain

$$
a_{m+1}^{-[1(1-r, m)]} a_{m+2}^{1\left\{(1-r / m)\left[1-r_{1}(m+1)\right]\right\}}<K^{1\left\{(1-r / m)\left[1-r^{\prime}(m+1)\right]^{1}\right.} .
$$

Next, writing $n=m+2$, and so on, we obtain a number of inequalities of which, if we take $p$ of them, the last is

$$
a_{m+p-1}^{-\left[1 /\left\{\prod_{t=0}^{m-2}\left(1-\frac{r}{m+t}\right)\right\}\right]} \underset{a_{m+p}}{1 /\left\{\prod_{t=0}^{p-1}\left(1-\frac{r}{m+t}\right)\right\}}<K^{1} /\left\{\prod_{t=1}^{p-1}\left(1-\sum_{m+t}^{r}\right)\right\} .
$$

Multiplying these inequalities together, we obtain

$$
a_{m}^{-1} a_{m+p}^{1 /\left\{\prod_{t=0}^{n-1}\left(1-{ }_{m+t}^{r}\right)\right\}}<K^{S_{p-1}} \text {.* }^{n}
$$

Consequently, if we multiply along (i.) by $\alpha_{m}$ and raise each side to the power $\eta_{p}$, we have

$$
\alpha_{m+p}^{1,(m+p)}<\alpha_{m}^{\eta_{1},} K^{\left(1-m \eta_{p}\right)(r+1)}
$$

Now, when $p$ is increased indefinitely the right-hand side tends to the limit $K^{1 /(r+1)}(r+1>0)$. Moreover, $1>K^{1 /(r+1)} \xi$; so that we can choose a positive number $\theta$ satisfying the inequalities

$$
1>\theta>K^{1(r+1)} \xi \text {. }
$$

It follows, then, that we can choose a number $P$ such that, if $p \geqslant P$, we have

$$
a_{n i}^{\eta_{\prime \prime}} K^{-[m(r+1)] \eta_{\prime \prime}} \leqslant \theta / K^{1 /(r+1)} \xi,
$$

since the right side is $>1$.

We have therefore $(p \geqslant P)$

$$
\alpha_{m+j^{\prime}}^{1^{\prime}(m+p)} \xi \gtrless \theta .
$$

In other words, if $m+P=N$, we have

$$
a_{n} \xi^{n}<\theta^{n},
$$

provided that $n \geqslant N$. Since $\theta$ is a proper fraction, the tirst part of the theorem is proved.

16. The proof of (2) is quite similar, and we will content ourselves with sketching it. It is sufficient to show that $P(z)$ diverges for values of $z$ such that $\xi>l^{-1 \cdot(r+1)}$. For any such value of $\hat{\xi}$ we can choose $\Omega$ number $k$ such that

$$
l>k>\xi^{-(r+1)} \text {. }
$$


It is then possible to choose a positive integer $m$ such that, if $n>m$, we have

$$
\frac{\alpha_{n+1}}{\alpha_{n}^{(1-r, n)}}>k \text {. }
$$

From this it easily follows that we can find a fixed number $\sigma>1$, such that a corresponding positive integer $N$ can be found in order to make

provided $n \geqslant N$.

$$
u_{n} \dot{\xi}^{n} \geqslant \sigma^{n} \text {, }
$$

This, of course, proves that the series diverges for all values of $z$ whose modulus is $\xi$. The theorem is then completely established.

Cor. I.-Writing $r=0$, we have Pincherle's theorem.

Cor. II.-If the set be such that there is only one limiting point, i.e., $l=L$, then

$$
\rho=L^{-1(r+1)} \text {. }
$$

